\title{
R Coronae Borealis stars
}

\author{
Ljiljana Skuljan, Peter L. Cottrell \\ Department of Physics and Astronomy, University of Canterbury, \\ Private Bag 4800, Christchurch 8020, New Zealand
}

\begin{abstract}
In the last ten years a significant step forward has been made toward a better understanding of the evolutionary status and unusual nature of the $\mathrm{R}$ Coronae Borealis (RCB) stars, a rare class of hydrogenpoor and carbon-rich variable stars. More detailed abundance analyses of the majority of RCB stars, and objects related to them, have become available in the last couple of years. In addition to this, recent theoretical studies of the most popular evolutionary models ("Double Degenerate" and "Final Flash") provide a new insight into the origin of these stars. Regarding the nature of the RCB declines, more observations from the light maxima and the decline phase are now available, including more data from space. However, the characteristics of the various emission lines appearing during the RCB declines, and the nature of their emitting regions, are still not entirely understood.
\end{abstract}

\section{Introduction}

The prototype of one of the exotic class of variable stars, $R$ Coronae Borealis (RCB), was discovered more than two hundred years ago. However, only over the last twenty years have observations covering the decline phase of a number of RCB stars been collected, which provide an opportunity for a more detailed study. The last general review of RCB stars was published seven years ago (Clayton 1996). Since then, there have been a number of interesting results concerning both the evolutionary status of the RCB stars and the nature of their declines.

The $\mathrm{R}$ Coronae Borealis stars are well known for their sudden and unpredictable declines occurring at irregular intervals. The stars decline rapidly by up to eight magnitudes in a few weeks. The recovery to normal light is usually significantly slower and may take months or even years. During these events complex spectroscopic (with a number of sharp and broad emission lines) and photometric changes have been observed.

It is generally accepted that RCB declines are caused by the obscuration of the stellar photosphere by a carbon dust cloud (Loreta 1934). This model is well supported by three facts: the shape of the light curve; the evolution of the emission lines; and the IR excess observed in all RCB stars. However, the triggering mechanism for the dust cloud condensation and the origin of various emission lines are not entirely explained by the dust cloud model. There are some alternative models (Clayton et al. 1997; Rao et al. 1999; Rosenbush 1998) 
proposed to interpret the unusual nature of the RCB stars and different aspects of their declines. Nevertheless, the dust cloud model is still the most accepted, and our focus will be mainly on the interpretation of the emission lines using this model.

In this review, the most important recent results concerning two aspects of the RCB stars, their evolutionary status and the origin of the emission lines, will be summarised.

\section{Evolution of $\mathbf{R}$ Coronae Borealis stars}

Even though the R Coronae Borealis stars are located in the post-AGB region on the HR diagram (Fig. 1), they cannot be considered as stars coming directly from this phase. Their chemical composition cannot be explained through the usual nucleosynthesis and mass loss characterizing this stage of evolution. In addition, the wider range of absolute magnitudes and a quite different population have to be taken into account if we include the newly-discovered RCB stars in the LMC (Alcock et al. 2001).

Since no reliable parallaxes have been measured for the galactic RCB stars (Cottrell \& Lawson 1998), the absolute magnitude of the class was based on three warm RCBs observed in the LMC (Feast 1979). With the most recent results from the MACHO database (Alcock et al. 2001), 13 RCBs have been discovered in the LMC, with a range of absolute magnitudes between -2.5 for the cool ones and -5 for the warm RCBs. The relative population of these stars in the LMC, 7:2:1 for $T_{\text {eff }} 5000: 6000: 7000 \mathrm{~K}$, appears to be quite different from the galactic ratio of 1:1:4 (Alcock et al. 2001). The LMC ratio, which is closer to that proposed theoretically (Weiss 1987; Lawson et al. 1990), implies that more RCB stars belong to the fainter or cooler population than to the warmer, more luminous, one. Therefore, the most recent results (Alcock et al. 2001) suggest that, if the population of the RCB stars in the two galaxies are similar, many more RCBs are expected in our galaxy (as many as 3200) compared to the $\sim 35$ currently known.

There have been two popular evolutionary models proposed for the RCB stars: FF - Final He-shell Flash model (Renzini 1990); and DD - Double Degenerate model (Webbink 1984). In both cases the RCB stars are considered to be new-born supergiants (Fig. 1), either through the final helium-shell flash scenario (FF model) or through the merging process of a $\mathrm{C}-\mathrm{O}$ and He white dwarfs (DD model).

The FF model implies connection with a planetary nebula, which has been detected in two RCB stars (UW Cen and R CrB, Pollacco et al. 1991). Three final-flash stars (V605 Aql, FG Sge and Sakurai's object) have been observed with the chemical compositions and the decline characteristics of the cool RCBs. In spite of some observational evidence in support of the FF model, rapid evolution of the final-flash stars (like V605 Aql) suggests a short lifetime for the RCB stars, inconsistent with the observations. However, the largest objection against the FF model comes from the predicted abundances. Only two incomplete theoretical studies of the FF model exist in the literature (Iben \& MacDonald 1995; Herwig et al. 1999). These models have not been able to explain the observed 


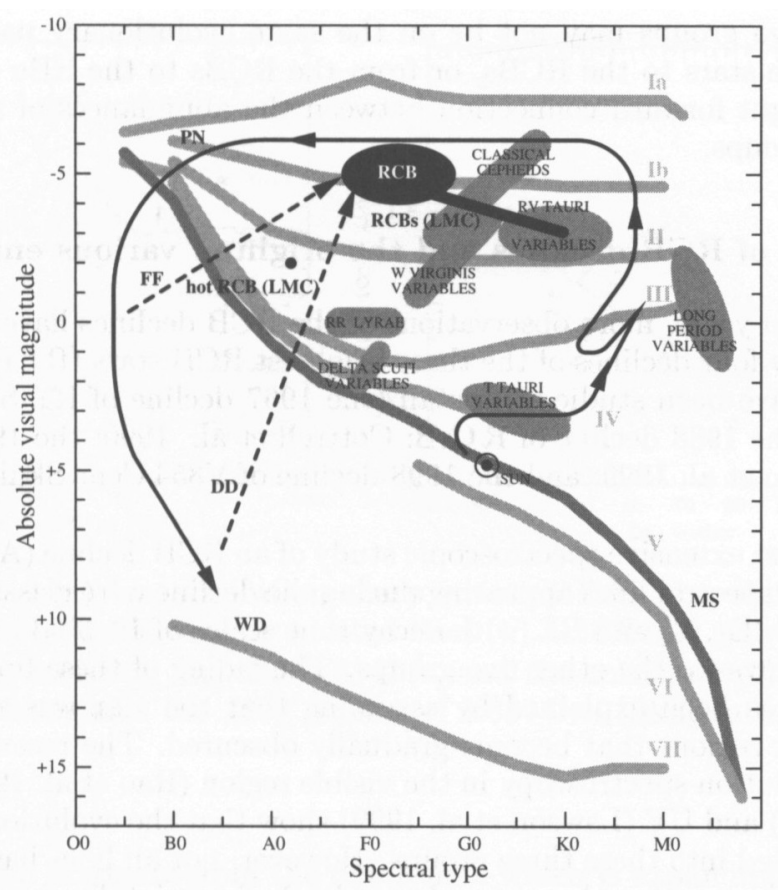

Figure 1. RCB stars on the HR diagram including RCBs from the LMC. Two possible evolutionary models are marked as FF and DD.

$\mathrm{C} / \mathrm{He}$ ratio, and the high ${ }^{12} \mathrm{C} /{ }^{13} \mathrm{C}$ ratio, as well as the anti-correlation between the abundances of $\mathrm{H}$ and $\mathrm{Fe}$.

It is important to mention that the most recent abundance analysis of 17 RCB stars (Asplund et al. 2000), using line-blanketed model atmospheres, shows clearly that most of the RCB stars exhibit a quite homogeneous composition. The minority group, characterized by a higher abundance of $\mathrm{H}$ compared to the majority group, and also lower metallicity, shows more scatter among its members.

The DD model naturally explains a lack of binarity among RCB stars and gives a longer lifetime than the FF model. However, the nuclear processes that can occur during the merging scenario, and how hydrogen can survive this process, are not well explained. The most recent theoretical analysis of the merging process of a $0.6 \mathrm{M}_{\odot} \mathrm{C}-\mathrm{O}$ white dwarf and a $0.3 \mathrm{M}_{\odot}$ He white dwarf can successfully reproduce most of the observable parameters of the extreme-helium (EHe) stars (Saio \& Jeffery 2002). RCB stars are usually considered to be related to the other two groups of hydrogen-deficient objects: the $\mathrm{HdC}$ and the EHe stars. The EHe stars are much hotter $(\sim 20000 \mathrm{~K})$ than the RCBs and, with the exception of three RCB-like stars, do not show declines and an IR excess. Some of them are also spectroscopic binaries, while no binarity is detected among the RCB stars. However, a detailed abundance analysis by Pandey et al. (2001) of the cooler EHe stars $\left(T_{\text {eff }} \sim 13000 \mathrm{~K}\right)$, which overlap the hotter RCBs, shows 
that these two groups may not be on the same evolutionary path. Evolution from the EHe stars to the RCBs, or from the RCBs to the EHe stars does not allow a straight forward connection between the abundances of some elements of the two groups.

\section{Nature of RCB declines and the origin of various emission lines}

In the last ten years, more observations of the RCB declines have become available, but only four declines of the three brightest RCB stars (R CrB, RY Sgr and V854 Cen) have been studied in detail (the 1967 decline of RY Sgr: Alexander et al. 1972; the 1988 decline of R CrB: Cottrell et al. 1990; the 1995-96 decline of R CrB: Rao et al. 1999; and the 1998 decline of V854 Cen: Skuljan \& Cottrell 2002).

In the first extensive spectroscopic study of an RCB decline (Alexander et al. 1972), all the spectral lines appearing during the decline were classified into three major groups: $E_{1}, E_{2}$ and $B L$, with decay time scales of 10-30 days for $E_{1}$ group and 50-150 days for the other two groups. The fading of these lines throughout the decline has been explained by assuming that the star was surrounded by three distinct regions that become gradually obscured. The recent results from the high resolution spectroscopy in the visible region (Rao et al. 1999; Skuljan \& Cottrell 2002) and UV (Lawson et al. 1999) show that the evolution of most lines can be classified into these three groups. However, not all lines have to originate from the above mentioned regions. Large intrinsic variability between declines, which is not consistent with this simple model, was observed for V854 Cen in the UV region (Lawson et al. 1999).

Lines can be classified into sharp and broad emission lines, as well as highvelocity absorption. Sharp emission lines include a number of low-excitation lines $\left(\chi_{u} \leq 5 \mathrm{eV}\right)$ : multiplets of BaII, Sc II, TiII, singly ionised and neutral metals (Fe I, Fe II, Ni I) and components of the complex line profiles of $\mathrm{NaI} \mathrm{D}$ and $\mathrm{Ca}$ II IR triplet. The high-excitation lines $\left(\chi_{u} \geq 10 \mathrm{eV}\right)$ of CI and OI, observed in some declines during the initial stage, are also sharp lines. Molecular bands $\left(\mathrm{C}_{2}\right.$ and $\mathrm{CN}$ ) and forbidden lines ([O I], [CI], [Ca II], [N II]) sometimes show sharp emission components, while in other declines only broad features are detected. The broad emission lines ( $\mathrm{NaI} \mathrm{D,} \mathrm{H} \alpha, \mathrm{Ca} I \mathrm{H} \& \mathrm{~K}$ and CaII IR triplet), with FWHM of $\sim 300 \mathrm{~km} \mathrm{~s}^{-1}$, are typical features of the RCB declines.

Blue shifted Na I D absorptions, with velocities of up to $400 \mathrm{~km} \mathrm{~s}^{-1}$, and with a characteristic evolution observed during the recovery phase (Skuljan \& Cottrell 1999, 2002), are also common features of all RCB declines. These optically-thin absorption lines indicate the existence of high-velocity gas dragged with the dust cloud during the process of obscuration.

\subsection{Sharp line emitting regions}

In addition to the well known characteristics of the low-excitation lines (blueshifted by a few $\mathrm{km} \mathrm{s}^{-1}$ and with a decay time scale of the $E_{2}$ group), the new observations (Rao et al. 1999; Skuljan \& Cottrell 2002) show that these lines have a multicomponent structure (Fig. 2a). The central component is the strongest while two broader side components are weaker and centred on the photospheric velocity. Detailed analysis of these lines indicates that the side 

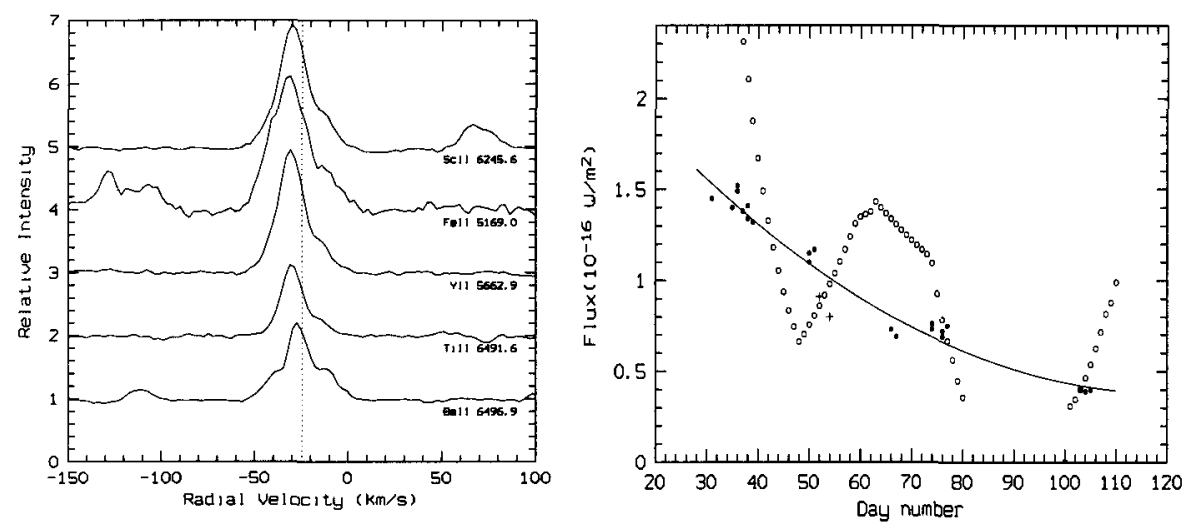

Figure 2. (a) Low-excitation sharp emission lines blue-shifted relative to the systemic velocity (dotted line) (b) Line fluxes (filled circles) of a sharp emission line, BaII $6142 \AA$, and the continuum flux (open circles) throughout the 1998 decline of V854 Cen (Skuljan \& Cottrell 2002).

components can originate from the outer shell which is expanding faster, while the central one is coming from the slower-moving inner parts of the same region. Similar excitation temperatures $(\sim 6000 \mathrm{~K})$, slightly cooler than the photospheric value, were found for these regions. The flux distribution of a number of the low-excitation lines were studied during the 1998 decline of V854 Cen (Skuljan \& Cottrell 2002). It was shown that the line fluxes change about ten times slower than the photospheric flux and stay almost constant during the initial stage of the decline (Fig. 2b). The position of this emitting region was estimated to be between 3 and $5 R_{*}$. This was deduced from the flux decay timescale and the acceleration of the dust cloud using the high-velocity $\mathrm{NaI} \mathrm{D}$ absorption and assuming that the dust cloud was formed close to the star (at $\sim 2 R_{*}$ ).

Red-shifted high excitation lines of $\mathrm{CI}$ and $\mathrm{OI}$ have been observed during the initial phases of two declines (Rao et al. 1999; Skuljan \& Cottrell 2002). These lines are optically thick and have a single component structure. They have been classified as transient emission lines (Rao et al. 1999) and observed prior to the decline onset as a disturbed photospheric absorption. This indicates that the high-excitation lines might originate in the photosphere and be related to the shock triggering the decline onset.

The lines from the $\mathrm{C}_{2}$ Swan system have been observed to have a blue-shift and excitation temperature of about $5000 \mathrm{~K}$, similar to the low-excitation lines. This suggests that they probably originate from the same region. On the other hand, absorption lines of the $\mathrm{C}_{2}$ Phillips system, detected during one decline of V854 Cen (Rao \& Lambert 2000), and with a rotational temperature of about $1100 \mathrm{~K}$, indicate much colder gas.

A schematic diagram of the various sharp-emission lines (Fig. 3a) shows that different emitting regions are involved in their production. While the lowexcitation lines of Sc II, Ti II and Na I D possibly originate from a region located 
$\mathbf{a}$

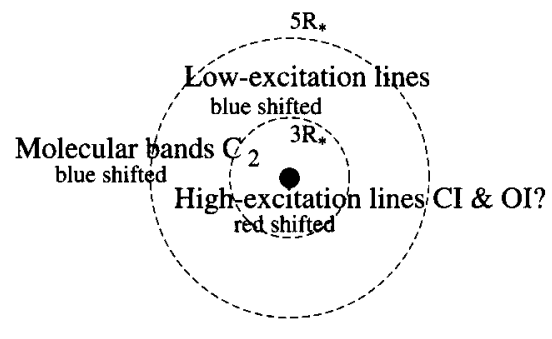

b

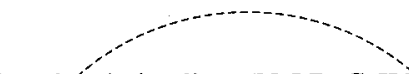

Broadémission lines ( $\mathrm{NaI} \mathrm{D}, \mathrm{CaII}$ 广̀ \& K).

permanent features; hot, dense, high-velocity region

High-excitation broad lines (HeI)?
Sharp forbidden lines (blue shifted)
Broad forbidden lines' (low-density nebula)

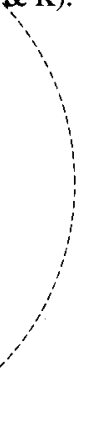

Figure 3. Schematic diagram of different emitting regions: (a) the sharp emission lines (see Section 3.1); and (b) the broad emission lines (see Section 3.2) Dotted circle represents the position of extended BL region at a few thousand $\mathrm{AU}$ from the star.

closer to the star, the forbidden lines, although having a similar blue-shift, are indicative of the more extended nebula. There are also some observations of the low-excitation lines at the light maximum, suggesting their permanent nature (Rao et al. 1999). On the other hand, the high-excitation red-shifted lines of C I and OI may originate in the photosphere.

\subsection{Broad line emitting regions}

The broad emission lines include high-excitation lines of He I, low-excitation lines ( $\mathrm{NaI} \mathrm{D,} \mathrm{Ca} \mathrm{II} \mathrm{H} \mathrm{\&} \mathrm{K),} \mathrm{as} \mathrm{well} \mathrm{as} \mathrm{forbidden} \mathrm{lines.} \mathrm{These} \mathrm{broad} \mathrm{emission}$ lines imply different possible sources of their excitation, emanating from different emitting regions. The source of the excitation for the high-excitation lines of $\mathrm{He} \mathrm{I}$ is not the same as for the low-excitation broad components, even though the lines have similar velocities. The most recent results show some of the interesting characteristics of these lines.

The 1992 decline of a hot RCB star, MV Sgr, demonstrates that the width of the high-excitation lines is greater than the width of the resonance and forbidden lines (see Fig. 11, Pandey et al. 1996). Although this was concluded for a hot RCB star, the analysis suggests that the gas closer to the star is moving faster than gas at greater distances. In addition, the flux ratio of the broad HeI spectral lines indicates a high-temperature $\left(\sim 2 \times 10^{4} \mathrm{~K}\right)$ and high-density $\left(N_{e} \sim 10^{11}\right.$ $\mathrm{cm}^{-3}$ ) emitting region, permitting collisional excitation (Rao et al. 1999). This is quite the opposite to the radiative source of the excitation for the low-excitation broad lines.

The most recent analysis of the $\mathrm{NaI} \mathrm{D}$ broad components (Rao et al. 1999; Skuljan \& Cottrell 1999, 2002) indicates some additional characteristics. Constant flux of the NaI D broad lines throughout the 1998 decline of V854 Cen, and the same intensity between two successive declines, suggests that these lines 
might be permanent features. The strongest broad emission was observed in V854 Cen, which exhibits very frequent declines. This indicates possible correlation between the frequency of the declines and the strength of the broad $\mathrm{NaI}$ $\mathrm{D}$ emission. In each of the declines a significant amount of the dust responsible for the broad emission has been formed. These results are supported by the new long-slit UV spectra of V854 Cen at light maximum (Clayton \& Ayres 2001). Broad C II $1335 \AA$ emission in V854 Cen was detected to be significantly extended from the star $(\sim 6000 \mathrm{AU})$. However, the BL lines are sometimes seen to fade significantly during the decline, indicating a position of this region closer to the star (Lawson et al. 1999).

Broad emission lines originate from a number of different regions (Fig. $3 b$ ). Broad forbidden lines are an indicator of the low-density nebula, while the other broad lines are more associated with the hot, dense and high-velocity gas. As we have seen, this region is probably a permanent feature of the RCB stars. The broad lines are also not affected by the dust cloud in the same way as the sharp lines and so it is likely that the broad lines are associated with a more extended region.

\section{Conclusion}

The most significant aspects of the $\mathrm{R}$ Coronae Borealis stars, their evolutionary status and the origin of various emission lines, are still not entirely understood. However, the most recent results have made an important contribution in understanding these two problems.

Abundance analyses of many RCB stars and related objects have been determined. An evolutionary model (Saio \& Jeffery 2002) for the merger of C-O and He white dwarfs into an EHe star is the first to reproduce nearly all parameters of the EHe stars. If RCBs are to be considered as the merger of two white dwarfs, then this model can account for the general properties of the RCB surface composition. However, it has to be acknowledged that the FF model is supported by some observational facts: nebulosity discovered around some RCBs and the observations of a few FF stars displaying RCB-like behaviour.

The dust cloud condensation model is still the most popular model of the $\mathrm{RCB}$ declines in spite of the fact that the $\mathrm{CO}$ molecules have not yet been detected and the origin of various emission lines have not been well explained. Weak CO absorption was detected in the far UV-spectra of RY Sgr with the Hubble Space Telescope by Clayton et al. (1999), but only at the light maximum. Observations of the $\mathrm{CO}$ bands during the decline events would be a real indicator of their importance in the dust cloud condensation.

The most recent observations of the various emission features suggest some new characteristics of these lines. The multicomponent structure, permanent nature and the flux distribution for the low-excitation lines, imply that the position of the emitting region is close to the star. On the other hand, the broad line emitting region is quite extended and not affected by the dust as shown with the most recent spectroscopic analyses and space observations. However, important questions about the source of the excitation for the high-excitation $\mathrm{He}$ I spectral lines and the position of their emitting region remains unsolved. 


\section{References}

Alcock, C., et al. 2001, ApJ, 554, 298

Alexander, J.B., Andrews, P.J., Catchpole, R.M., Feast, M.W., Lloyd Evans,

T., Menzies, J.W., Wisse, P.N.J., Wisse, M. 1972, MNRAS, 158, 305

Asplund, M., Gustafsson, B., Lambert, D.L., Rao, N.K. 2000, A\&A, 353, 287

Clayton, G.C. 1996, PASP, 108, 225

Clayton, G.C., Ayres, T.R. 2001, ApJ, 560, 986

Clayton, G.C., Bjorkman, K.S., Nordsieck, K.H., Zellner, N.E.B., Schulte-Ladbeck, R.E. 1997, ApJ, 476, 870

Clayton, G.C., Ayres, T.R., Lawson, W.A., Drilling, J.S., Woitke, P., Asplund M. 1999, ApJ, 515, 351

Cottrell, P.L., Lawson, W.A. 1998, PASP, 15, 179

Cottrell, P.L., Lawson, W.A., Buchhorn, M. 1990, MNRAS, 244, 149

Feast, M.W. 1979, in Changing trends in variable star research, IAU Coll. No. 46, eds. F.M. Bateson, J. Smak, \& I.M. Urch, (Hamilton, University of Waikato), 246

Herwig, F., Blocker, T., Langer, N., Driebe, T. 1999, A\&A, 349, L5

Iben, I., MacDonald, J. 1995, in White dwarfs, Proc. 9th European Workshop on White Dwarfs, eds. D. Koester \& K. Werner (Springer-Verlag, Berlin), 48

Lawson, W.A., Cottrell, P.L., Kilmartin, P.M., Gilmore, A.C. 1990, MNRAS, 247,91

Lawson, W.A., Maldoni, M.M., Clayton, G.C., Valencic, L., Jones, A.F., Kilkenny, D., van Wyk, F., Roberts, G., Marang, F. 1999, AJ, 117, 3007

Loreta, E. 1934, Astr. Nach., 254, 151

Pandey, G., Rao, N.K., Lambert, D.L. 1996, MNRAS, 282, 889

Pandey, G., Rao, N.K., Lambert, D.L., Jeffery, C.S., Asplund, M. 2001, MNRAS, 324,937

Pollacco, D.L., Hill, P.W., Houziaux, L., Manfroid, J. 1991, MNRAS, 248, 1P

Rao, N.K., Lambert, D.L. 2000, MNRAS, 313, L33

Rao, N.K., Lambert, D.L., Adams, M.T., Doss, D.R., Gonzalez, G., Hatzes, A.P., James, C.R., Johns-Krull, C.M., Luck, R.E., Pandey, G., Reinsch, K., Tomkin, J., Woolf, V.M. 1999, MNRAS, 310, 717

Renzini, A., 1990, in ASP Conf. Ser., Vol. 11, Confrontation Between Stellar Pulsation and Evolution, eds C. Cacciari \& G. Clementini, (ASP: San Francisco), 549

Rosenbush, A.E. 1998, Kinemat. Fiz. Nebesn. Tel., 14, 342

Saio, H., Jeffery, C.S. 2002, MNRAS, 333, 121

Skuljan, Lj., Cottrell P.L. 1999, MNRAS, 302, 341

Skuljan, Lj., Cottrell P.L. 2002, MNRAS, 335, 1133

Webbink, R.F. 1984, ApJ, 277, 355

Weiss, A. 1987, A\&A, 185, 178 


\section{Discussion}

Percy: 1) Could you comment on the pulsational behaviour of V854 Cen, which could be a possible triggering mechanism? 2) Could the DD and FF models both be correct, both producing RCB stars?

Skuljan: 1) V854 Cen has a regular pulsations, but no radial velocity variations have been detected indicating the shocks. Dust clouds still can condense (either close to, or farther from the star) if the right conditions are achieved (low temperature for the condensation of amorphous carbon $\mathrm{T} \sim 2000 \mathrm{~K}$ ). 2) Yes, it is possible. Observational evidence shows that Planetary Nebulae exist around a couple of RCB stars. This makes the connection with the FF model. It is possible that the FF model is responsible for the majority of RCBs, and the DD model is responsible for a minority of RCBs. However, this should be proved by theoretical prediction of the abundance analysis on detail models of FF and DD.

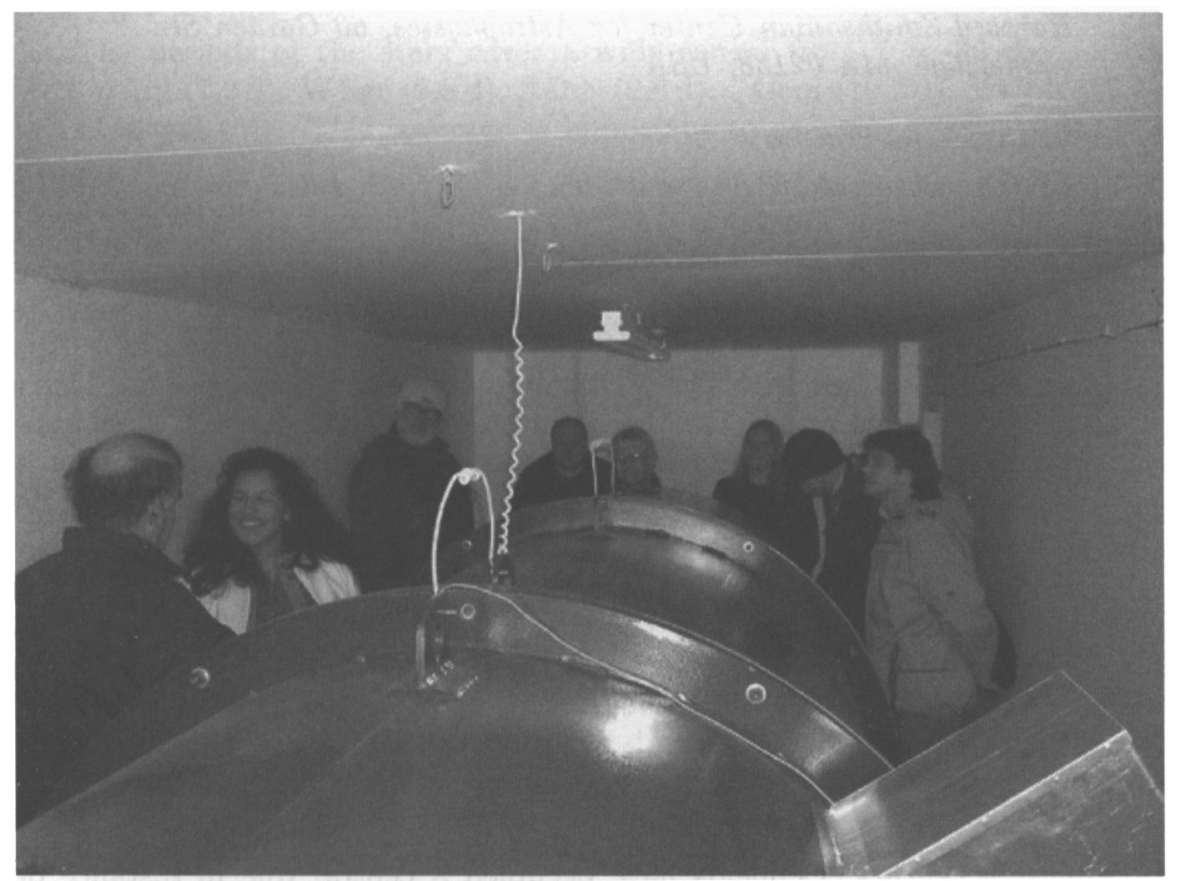

A tour of the Hercules Spectrograph at Mt John Observatory 